\title{
Production value optimization with intermediate jig product recirculation
}

\author{
Joachim Pielot ${ }^{1, \text { a }}$ \\ ${ }^{1}$ Silesian University of Technology, Department of Electrical Engineering and Control in Mining, 44-100 Gliwice, \\ ul. Akademicka 2, Poland
}

\begin{abstract}
The paper describes the result of the production value increase in systems with two and three jigs and recirculation of the interemediate product compared to the output value obtained from a single jig. The increase is greater when the reference ash content in the concentrate is lower.
\end{abstract}

\section{Secondary separation with a recirculation of the intermediate product}

Either a part or the whole intermediate product stream can again enter the input of the three-product separator or the system of two two-product separators. This layout is shown in Fig. 1. The rate of recirculation $R_{i p}$ determines which part of the intermediate product flow returns back to the input and is mixed with the input raw feed stream.

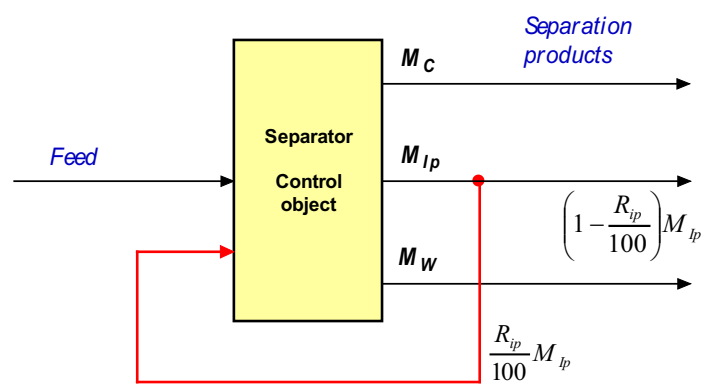

Figure 1. Three-product gravitational separator as a control object with either partial or complete recirculation of the intermediate product; $M_{C}, M_{I p}, M_{W}$ - concentrate, intermediate product and waste masses.

According to the concept shown in Fig. 1 it is possible to create two versions of separation with intermediate product recirculation, as illustrated in Fig. 2. Symbol $r W C$ means recirculation of intermediate fractions obtained from the portion of the waste fractions from the first separator and the lighter fractions from the second separator. The $r C W$ symbol means recirculation of intermediate fractions obtained from the part of the concentrate fraction part from the first separator and sinking

\footnotetext{
${ }^{\mathrm{a}}$ Corresponding author: Joachim.Pielot@polsl.pl
} 
fractions from the second separator. Recirculation coefficients $R_{i p 1}$ and $R_{i p 2}$ in both systems determine what part of the intermediate product stream goes back again to the separation process input.

a)

b)

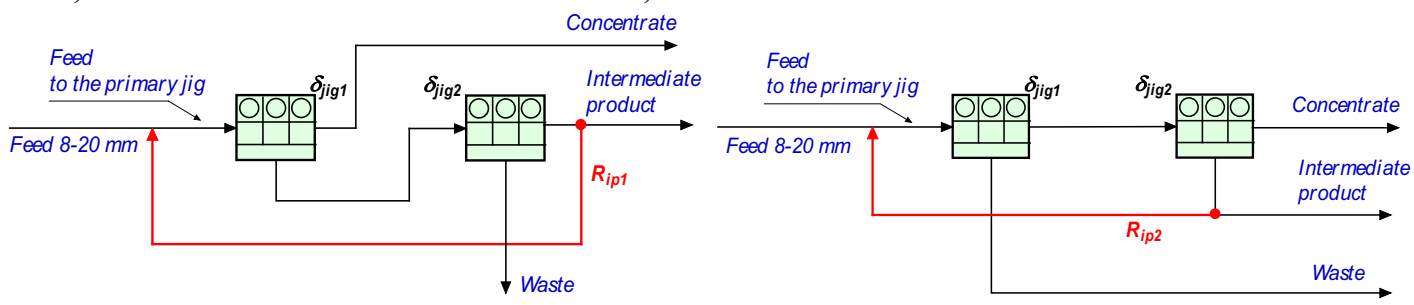

Figure 2. Systems of two two-product jigs with recirculation of an intermediate product: a) $2 j i g r W C$, b) $2 j i g r C W$

\section{Optimum control with product recirculation}

As the value of production there has been adopted a product of the concentrate mass and its qualitydependent unit price, as determined from the $4^{\text {th }}$ version of the sales formula of the year 2002 [6].

As a goal function of the maximization algorithm it has been assumed a value of the concentrate output:

$$
P V_{i}\left(\delta_{j i g 1}, \delta_{j i g 2}, R_{i p}\right)=M_{C i}\left(\delta_{j i g 1}, \delta_{j i g 2}, R_{i p}\right) \cdot P_{C i}\left(\delta_{j i g 1}, \delta_{j i g 2}, R_{i p}\right),
$$

which is maximized:

$$
\max _{\left(\delta_{j i g 1}, \delta_{j i g 2}, R_{i p}\right) \in R^{n}} P V_{i}\left(\delta_{j i g 1}, \delta_{j i g 2}, R_{i p}\right)
$$

with an equivalence restriction of the concentrate ash content:

$$
A_{C}=A_{C i},
$$

at subsequent $A_{c i}$ values of $6,9,12,15$ and $18 \%$ :

where: $P V$ - production value, PLN,

$\delta_{j i g}$ - jig separation density (decision variables of a production maximization algorithm), $\mathrm{g} / \mathrm{cm}^{3}$,

$R_{i p}$ - partial recirculation coefficient of the of the intermediate product, $\%$,

$M_{C}$ - concentrate mass, $\mathrm{Mg}$,

$P_{C i}$ - concentrate unit price, dependent on the coal quality, PLN/Mg [8].

All simulation forecasts were carried out using array models of jig separation curves [5]. There was included a hardly-washable feed with characteristics as in the paper [3].

Figure 3 illustrates obtained dependence of the relative production value in a $2 j i g ~ r W C$ layout. The reference level is different in each case (with different requested ash content values) maximum output value of production achieved with the desired concentrate ash content of the concentrate in a system without intermediate product recirculation. With the increase recirculation ratio of intermediate and taken into account the criterion of maximizing the objective function (2) the value of production increases. This is roughly equivalent to an increase in the production value with jig concentrate recirculation (Fig. 4) [3]. The advantage of the intermediate product recycling, however, is that the feed amount in this case grows very slightly (recirculate only grains with densities similar to the separation density). Increase in the feed amount of the order of a few percent is several times lower than in the case of concentrate recirculation. 


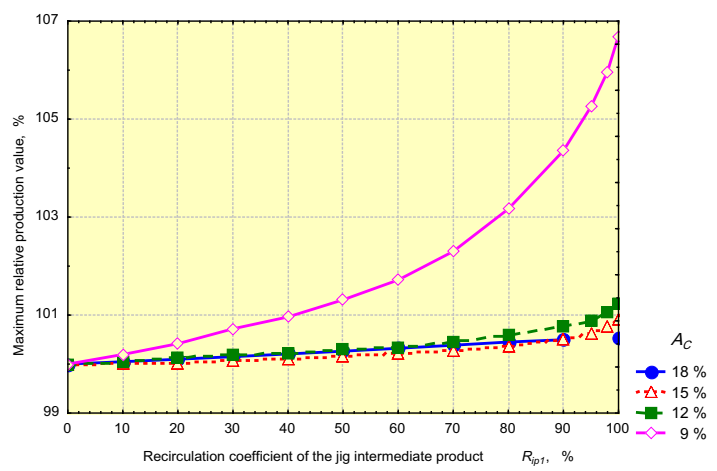

Figure 3. Maximum relative production value of a concentrate from jigs in system $2 \mathrm{jig} r W C$ at changes of recirculation coefficient of intermediate product $R_{i p 1}$.

Figure 4 shows an example of optimally selected separation densities in a $2 \mathrm{jig} r \mathrm{CW}$ system, at the lowest desired concentrate ash content value $-6 \%$, and at $12 \%$. As the value of the recirculation coefficient increases, there is a noticeable increasing difference between the chosen separation densities in both jigs.

a)

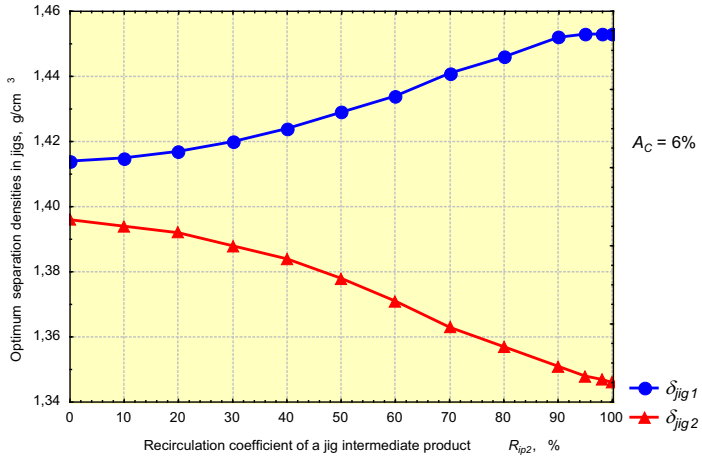

b)

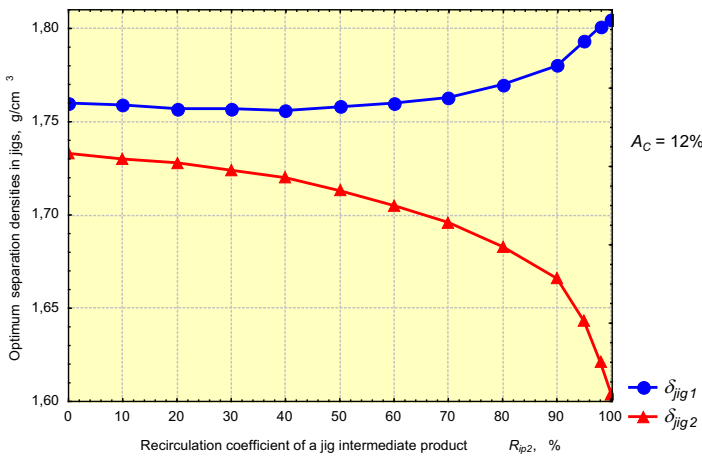

Figure 4. Optimum separation densities in both jigs in system 2jig $r C W$ at changes of recirculation coefficient of intermediate product $R_{i p 2}: A_{k}=6 \%$ (a), $A_{k}=12 \%(\mathrm{~b})$.

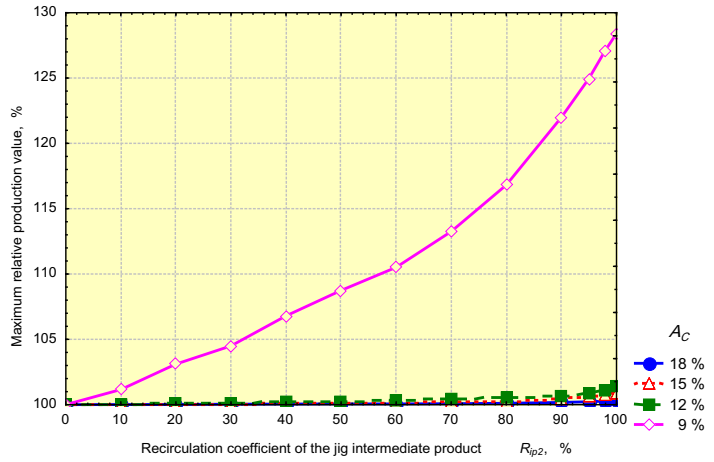

Figure 5. Maximum relative production value of a concentrate from jigs in system $2 \mathrm{jig} r \mathrm{CW}$ at changes of recirculation coefficient of intermediate product $R_{i p 2}$. 


\section{Comparison of the maximum output value in jig systems with intermediate product recirculation}

Output production values for two jig systems with intermediate product recirculation presented in Section 2 are given in relative terms, so it is not visible, where the absolute value of the system output is higher. In order to compare the value of production, the calculations were performed with desired concentrate ash content growing of $1 \%$.

Since the maximum value of output production can be obtained by the full recirculation of intermediate product (Figs. 3 and 5) further analysis was therefore limited to those cases $\left(R_{i p 1}=100 \%\right.$ or $R_{i p 2}=100 \%$ ). There was made a comparison of production values achieved in the four systems. The first system was a single jig (without recirculation), two more systems are presented in Fig. 2, and the fourth system was a group of three jigs with two completely recirculating intermediates, as shown in Fig. 6 . The maximum value of a single jig output production was a reference level (100\%).

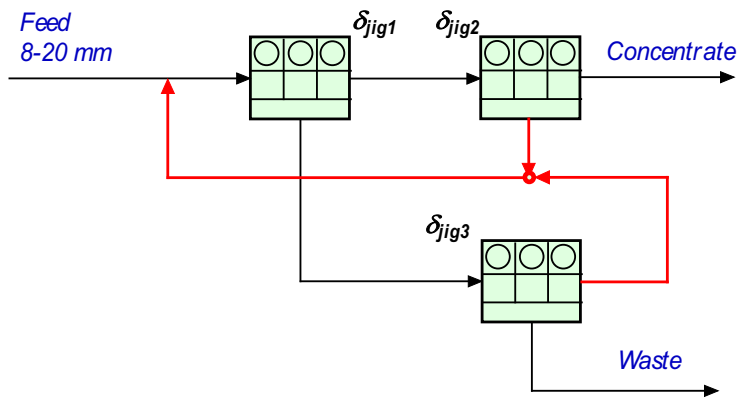

Figure 6. System of three two-product jigs 3 os $2 r$ with recirculation of two intermediate products.

The objective function of the maximization algorithm is a concentrate production value. For one jig:

$$
V P_{i}\left(\delta_{j i g}\right)=M_{C i}\left(\delta_{j i g}\right) \cdot P_{C i}\left(\delta_{j i g}\right)
$$

In the case of the two-jig systems shown in Fig. $2\left(R_{i p l}=100 \%\right.$ or $\left.R_{i p 2}=100 \%\right)$ :

$$
V P_{i}\left(\delta_{j i g 1}, \delta_{j i g 2}\right)=M_{C i}\left(\delta_{j i g 1}, \delta_{j i g 2}\right) \cdot P_{C i}\left(\delta_{j i g 1}, \delta_{j i g 2}\right) .
$$

In the case of the three-jig circuit shown in Fig. 6:

$$
V P_{i}\left(\delta_{j i g 1}, \delta_{j i g 2}, \delta_{j i g 3}\right)=M_{C i}\left(\delta_{j i g 1}, \delta_{j i g 2}, \delta_{j i g 3}\right) \cdot P_{C i}\left(\delta_{j i g 1}, \delta_{j i g 2}, \delta_{j i g 3}\right) .
$$

There are maximized functions (4), (5) and (6) at equivalence restrictions and at subsequently asked $1 \%$ concentrate ash content increments:

$$
A_{C}=A_{C i} .
$$

The calculation results are presented in Fig. 7. In each system with intermediate product recirculation there can be achieved higher production value than the production of the single-jig system. This is particularly important for small values of desired concentrate ash content. The highest production value is achieved in a three jig system 3 jig $2 r$, slightly lower production value can be achieved in a two-jig system 2 jig $r C W$. Comparing both two-jig systems it can be said that at a better desired concentrate quality higher production value can be provided by a 2 jig $r C W$ system p (Fig. 2b), and at worse quality - 2 jig $r W C$ system (Fig. 2a). 


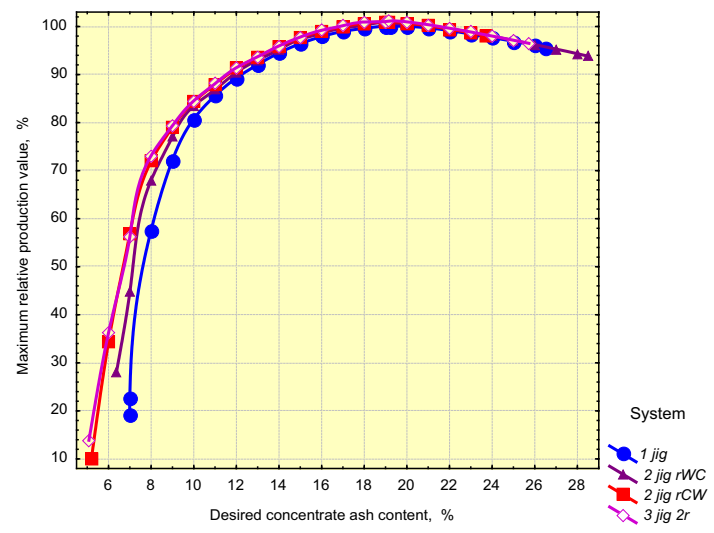

Figure 7. Maximum relative production value of a concentrate from one jig and in three systems at complete recirculation of an intermediate product.

\section{Summary}

In any system with intermediate product recirculation the output value is higher than the output value of a single separator. The increase in a production value is more significant when the reference concentrate ash content value is lower. Therefore, the application of intermediate product recirculation is profitable for investment and operation [4].

A comparison of the three jig separation systems shows that the highest output value is achieved in a 3 jig $2 r$ system with three jigs, slightly lower production value can be achieved by a 2 jig $r C W$ layout. Comparing the two systems with two jigs it can be said that with a better set point of concentrate quality higher output value (Fig. 7) can be achieved in a $2 j i g r C W$ system (Fig. 2b), while at the lower concentrate quality the 2 jig $r W C$ system (Fig. 2a) provides higher output value.

Gravity separators are characterized by a certain separation inaccuracy enrichment. It is a result of the fact that the separation curves have a shape deviating from the ideal curve. The use of secondary separation with a recirculation of the intermediate product allows for the effect equivalent to the separation curve shape improvement of a single separator [8].

\section{References}

1. W. Blaschke, Wydawnictwo IGSMiE PAN (Krakow, 2009)

2. J. Blaschke (i in.), IM, 4(17) (2006)

3. S. Boron, A. Heyduk, J. Pielot, in Proceedings of Mineral Engineering Conference, MEC (2016)

4. S. Boron, J. Pielot, A. Wojaczek, Miner. Resour. Manag., 30(2), 67-82 (2014)

5. F. Goodman, J. McCreery, Coal Prep. Compu. Model, 112 (1980)

6. U. Lorenz, W. Blaschke, Z. Grudzinski, Monograph (Wydawnictwo IGSMiE PAN, Krakow, 112, 2002)

7. J. Pielot, Monograph, (Wydawnictwo Politechniki Slaskiej, 306, 2011)

8. J. Pielot, in Proceedings of Mineral Engineering Conference MEC 2015, (Szczawnica, 24, 2015) 